\title{
56.6 dB high gain L-band EDFA utilizing short-length highly-doped erbium rare-earth material
}

\author{
M. H. Al-Mansoori \\ mmansoori@Soharuni.edu.om
}

W. S. Al-Ghaithi
Faculty of Engineering, Sohar University, PO Box 44, PCI 311, Sohar, Oman

Faculty of Engineering, Sohar University, PO Box 44, PCI 311, Sohar, Oman

In this paper, we experimentally investigate the performance of an efficient high gain L-band erbium-doped fiber (EDF) amplifier structure utilizing short-length highly-doped erbium rare-earth material with a single pump source. The amplifier gain and noise figure variation for different amplifier structures have been investigated. A filter is used to reduce the self-saturation effect and suppress the C-band amplified spontaneous emission (ASE) noise. The amplifier achieves a signal gain of $56.6 \mathrm{~dB}$ with a low noise figure of $4.8 \mathrm{~dB}$ at $-50 \mathrm{dBm}$ input signal power using only $8 \mathrm{~m}$ of EDF length. The amplifier gain shows significant improvement of $6 \mathrm{~dB}$ with $\mathrm{C} / \mathrm{L}$ band coupler and $13 \mathrm{~dB}$ with tunable-band pass filter compared to amplifier structure without ASE suppression.

[DOI: http://dx.doi.org/10.2971/jeos.2014.14028]

Keywords: Optical amplifiers, EDFA, L-band, optical filters, optical networks

\section{INTRODUCTION}

Erbium Doped Fiber Amplifiers (EDFAs) are one of the most efficient optical fiber amplifiers in the present and future development of all fiber-to-the home (FTTH) optical networks and long-haul dense wavelength division multiplexed (DWDM) optical communication systems [1]. It operates in a wide band covering C-band (conventional band) with wavelength range from $(1530-1565) \mathrm{nm}$ and L-band (long wavelength band) with wavelength range from $(1570-1610) \mathrm{nm}$ [2]-[4]. However, the gain coefficient in the L-band window is smaller than that in the C-band, higher pump power and longer length of the doped fiber are normally used with L-band EDFA [5]. In literature, various gain enhancement techniques have been proposed to enhance the gain of L-band EDFA such as using narrow-band end-reflectors to feed a small fraction of the amplified spontaneous emission (ASE), up to a few tenths of a milliwatt, back into the EDF [6], utilizing unwanted backward ASE as a secondary pump source for the un-pumped section of erbium-doped fiber (EDF) [7], placing an optical circulator between two EDF segments in a two-stage reflective-type amplifier structure [8], utilizing a bidirectional pumping structure via inserting a $980 \mathrm{~nm}$ pump laser diode in the input part [9], incorporating a fiber Bragg grating (FBG) to reflect part of the C-band backward ASE into the EDF [10]-[13] or tunable band-pass filter in a double-pass configuration [14], adding a short length of forward pumped EDF in front of a double-pass amplifier [15], using loop configuration and a C-band seed signal [16]. Among the other gain enhancement techniques proposed is the use of a two stage structure with a bypass isolator [17] or in-line FBG with forward-backward pumping scheme [18], a three-stage structure of EDF pumped by $980 \mathrm{~nm}$ and $1480 \mathrm{~nm}$ laser diodes, which can achieve $35 \mathrm{~dB}$ gain with $5 \mathrm{~dB}$ noise figure [19] and the use of residual pump power in a three-stage configuration [20]-[21]. However, for a multistage EDFA configuration, the ASE keeps on increasing at each stage, hence reducing the overall gain of the system. Thus, the main limitation to the performance of multistage L-band EDFA is imposed by amplified spontaneous emission (ASE) noise. These limitations can be overcome by incorporating optical filter within the amplifier to suppress the ASE. In a recent work, a flat gain bandwidth of $17 \mathrm{~dB}$ in two-stage L-band EDFA using pump distribution technique and $25 \mathrm{~m}$ of EDF length is demonstrated [22].

Even though these approaches have enhanced the gain of Lband EDFA, the enhancement is at the expense of more than one pump source, longer EDF lengths and the high number of optical components used in the amplifier structure. Also, the amplifier gain was enhanced using optical filter inside the amplifier structure to suppress the ASE noise. To date, no published work on the effect of optical filter types on the amplifier gain has been reported. Therefore, it is desirable to design an efficient L-band EDFA with high gain and low noise figure using single pump source and short gain medium for simple integration with passive optical devices as well as the economic feasibility.

In this paper, we present experimental results that demonstrate an efficient triple-pass high gain L-band erbium-doped fiber amplifier. We compare experimentally the gain and noise figure characteristics of the amplifier at different optical filter. It is shown that the type of the optical filter play a significant role in obtaining a high gain optical amplifier. The amplifier gain of $56.6 \mathrm{~dB}$ with noise figure less than $4.8 \mathrm{~dB}$ using single pump source and short EDF length is achieved. To the best of our knowledge, this is the highest gain obtained in the development of L-band EDFA using a single pump source with only $8 \mathrm{~m}$ of EDF length. The high gain obtained is attributed to 


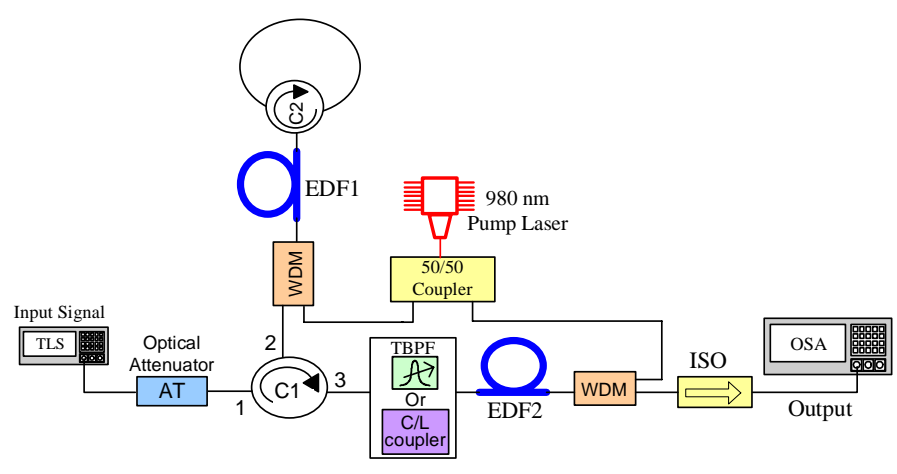

FIC. 1 Experimental setup of triple-pass L-band erbium-doped fiber amplifier with a single pump source.

the optimization of the pump powers, the input signal power and wavelengths and to the optical filter which suppress the amplified spontaneous emission noise.

\section{EXPERIMENTAL SETUP AND OPERATION PRINCIPLE}

Figure 1 shows the experimental setup of triple-pass L-band EDFAs, which consists of input laser source, two optical circulators $(\mathrm{C} 1, \mathrm{C} 2)$, pump laser, wavelength division multiplexer (WDM), two erbium doped fibers (EDF1,EDF2), $3 \mathrm{~dB}$ optical coupler, an optical isolator (ISO), and optical filter. A $980 \mathrm{~nm}$ pump laser is used to pump the gain medium with fifty percent of the pump power is distributed to the first stage while the remaining portion of pump power is distributed to the second stage by using a $3 \mathrm{~dB}$ optical coupler as depicted in Figure 1 . The length of the gain medium in the first stage and second stages are $5 \mathrm{~m}$ and $3 \mathrm{~m}$, respectively. The EDF1 and EDF2 have an absorption coefficient of $41.14 \mathrm{~dB} / \mathrm{m}$ at $1530 \mathrm{~nm}$, absorption of $23.9 \mathrm{~dB} / \mathrm{m}$ at $979 \mathrm{~nm}$, cut-off wavelength around $900 \mathrm{~nm}$ and mode field diameter of $5.8 \mu \mathrm{m}$.

The first stage of the amplifier is pumped in forward direction while the second stage is pumped in backward direction through a WDM which is used to multiplex the $980 \mathrm{~nm}$ pump power and the signal power. The ISO is placed at the output port to eliminate any back reflection. An optical filter (tunable band pass filter or C/L coupler) is inserted after port 3 of the first circulator (C1) to filter out the C-band backward amplified spontaneous emission (ASE) noise from saturating the gain of the second stage. The first circulator is used to launch the original input signal to the first stage. The second circulator (C2) is used as a fiber reflector which reflects the amplified signal at the end of first stage. The amplified optical signal is reflected at the fiber reflector and returns to EDF1 for double-pass amplification. The input signal is then passed to EDF2 through port 3 of C1 for triple-pass amplification. The $980 \mathrm{~nm}$ pump laser is varied from $105 \mathrm{~mW}$ to $405 \mathrm{~mW}$ in step of $50 \mathrm{~mW}$ while the input signal power is varied from -50 to $0 \mathrm{dBm}$ with different wavelengths. The amplifier gain and noise figure are taken at the output port of the ISO as shown in Figure 1 and are measured by an optical spectrum analyzer (OSA). The performance of the amplifier structure is investigated with and without including the filtering mechanisms.

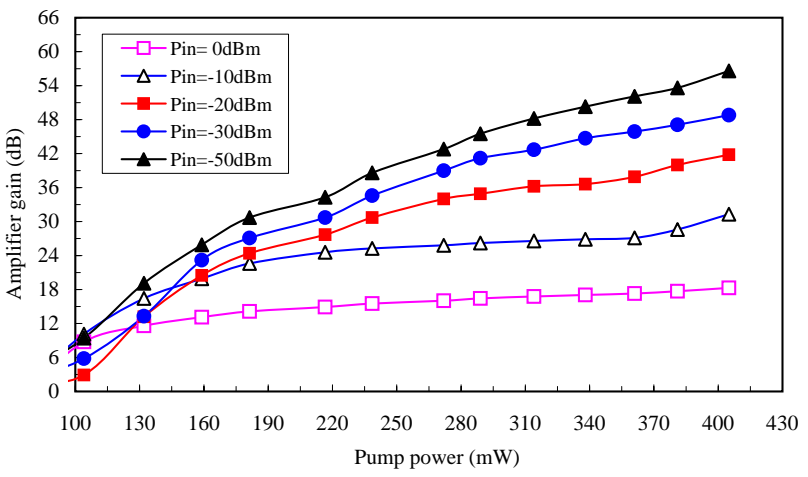

FIG. 2 Amplifier gain against $980 \mathrm{~nm}$ pump power at different input signal powers for $1570 \mathrm{~nm}$ signal wavelength.

\section{RESULT AND DISCUSSION}

The amplifier gain and noise figure depends on input signal power, wavelengths, length of the gain medium, pump powers and amplifier structure. To get high amplifier gain, we used optical filtering mechanism after the first stage to suppress the backward ASE noise from saturating the gain of the second stage. Figure 2 shows the effect of $980 \mathrm{~nm}$ pump powers on the amplifier gain at different input signal powers of $0 \mathrm{dBm},-10 \mathrm{dBm},-20 \mathrm{dBm},-30 \mathrm{dBm}$ and $-50 \mathrm{dBm}$. The wavelength of the input signal is fixed at $1570 \mathrm{~nm}$.

From this Figure, we can see that the gain of the EDFA increases with the increasing of the $980 \mathrm{~nm}$ pump power and then goes to saturation after a certain level of the pump power. At the saturation region, the increase in the gain becomes smaller due to the high power that provides population inversion for all the erbium ions in the fiber. In this region any increase on the $980 \mathrm{~nm}$ pump power does not contribute much to the improvement of gain as shown in Figure 2. For input signal power of $-50 \mathrm{dBm}$, the gain increases from $9.4 \mathrm{~dB}$ to $56.6 \mathrm{~dB}$ when the pump power increase from $105 \mathrm{~mW}$ to $405 \mathrm{~mW}$, respectively. This is because the erbium ion population inversion increases with the increment of EDF pump power. In addition, the amplifier gain is increased with the decrement of the input signal power.

The effect of the input signal wavelength on the amplifier gain is also investigated. Figure 3 shows the amplifier gain against input signal wavelength at different input power of $0 \mathrm{dBm}$ and $-50 \mathrm{dBm}$. The $980 \mathrm{~nm}$ pump power is fixed at $314 \mathrm{~mW}$. Within the tuning range of the tunable bandpass filter (TBPF), the input signal wavelength is varied from $1550 \mathrm{~nm}$ to $1600 \mathrm{~nm}$ in step of $5 \mathrm{~nm}$. At low input signal, the amplifier gain is a strong function of the signal wavelength due to the variation of the absorption and emission crosssections with wavelength [2]-[5]. For L-band, the signal at longer wavelengths experiences a lower gain in comparison to shorter ones. As is seen in Figure 3, the highest gain is obtained at $1570 \mathrm{~nm}$ input signal wavelength with power of $-50 \mathrm{dBm}$. This is due to the higher absorption and emission at $1570 \mathrm{~nm}$ wavelength. At longer wavelengths, the gain coefficient is reduced due to the low absorption and emission 


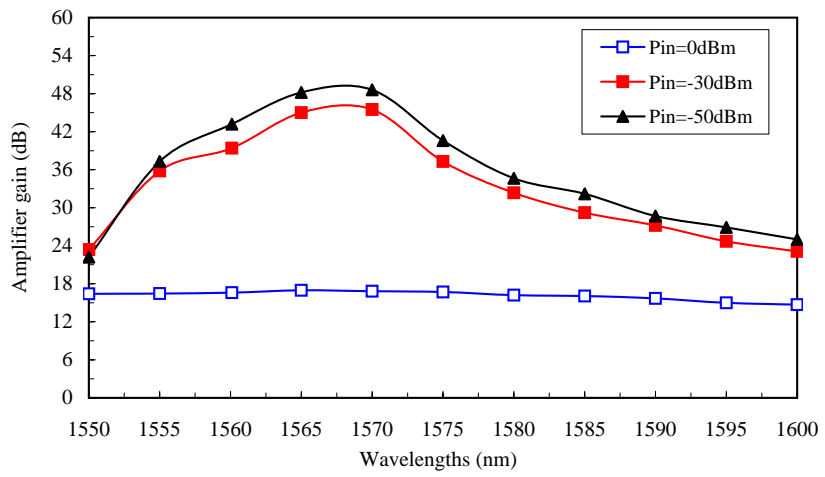

FIG. 3 Gain against input signal wavelength at $314 \mathrm{~mW}$ pump power with different input signal power.

coefficient. In addition, the amplifier gain is reduced with the increment of the input signal power.

The performance of the amplifier structure is also investigated at different input signal powers. Figure 4 shows the amplifier gain against input signal power at different EDF pump powers. The input signal wavelength is fixed at $1570 \mathrm{~nm}$. In this work, four different pump powers are applied and the signal power is increased from $-50 \mathrm{dBm}$ to $0 \mathrm{dBm}$. From Figure 4, it is seen that EDFA gain decreases with increasing signal input power. At $-50 \mathrm{dBm}$ input signal and $405 \mathrm{~mW}$ of $980 \mathrm{~nm}$ pump power a gain of $56.6 \mathrm{~dB}$ with noise of $4.8 \mathrm{~dB}$ is obtained. The population inversion level is dependent on the input signal power where the inversion is decreased as the input signal power is increased [7]. In addition, high input signal stimulates excited erbium-ions very fast and consequently the erbium-ions population depletes at the beginning of the fiber. Therefore, the pump power is unable to replenish the inversion of ions to the higher level as fast as the signal depleting the excited ions $[6,14]$. In Figure 4(b), the variation of noise figure is given as a function of input signal power for a fixed wavelength of $1570 \mathrm{~nm}$ and different EDF pump powers. In this experiment, the input signal power varies from $-50 \mathrm{dBm}$ to $0 \mathrm{dBm}$ in step of $-5 \mathrm{dBm}$.

The graph shows that the amplifier noise figure increases with increasing input signal power above $-10 \mathrm{dBm}$. For signal powers from $-40 \mathrm{dBm}$ to $-15 \mathrm{dBm}$, the noise figure is maintained. In addition, the noise figure decreases with increasing EDF pump powers. The noise figure of the amplifier varies inversely with the amplifier gain and linearly with ASE power. The high amplifier gain causes the spontaneous emission to stay in low levels. Since the backward C-band ASE noise was suppressed by the TBPF at the input of the the second stage, the noise figure is significantly reduced. As can be seen in Figure 4(b) at $1570 \mathrm{~nm}$ signal wavelength, the lowest noise figure of $4.8 \mathrm{~dB}$ is obtained at $405 \mathrm{~mW}$ pump power and $-50 \mathrm{dBm}$ input signal. These results indicates a better noise figure value than the $6 \mathrm{~dB}$ as reported in [6], $5 \mathrm{~dB}$ as reported in [12], $9.7 \mathrm{~dB}$ as reported in [16], and $5.5 \mathrm{~dB}$ as reported in [18].

The measured output spectrum by an optical spectrum analyzer (OSA) at the highest gain region is depicted in Figure 5. The recorded output power is $6.6 \mathrm{dBm}$ at $-50 \mathrm{dBm}$ input sig-
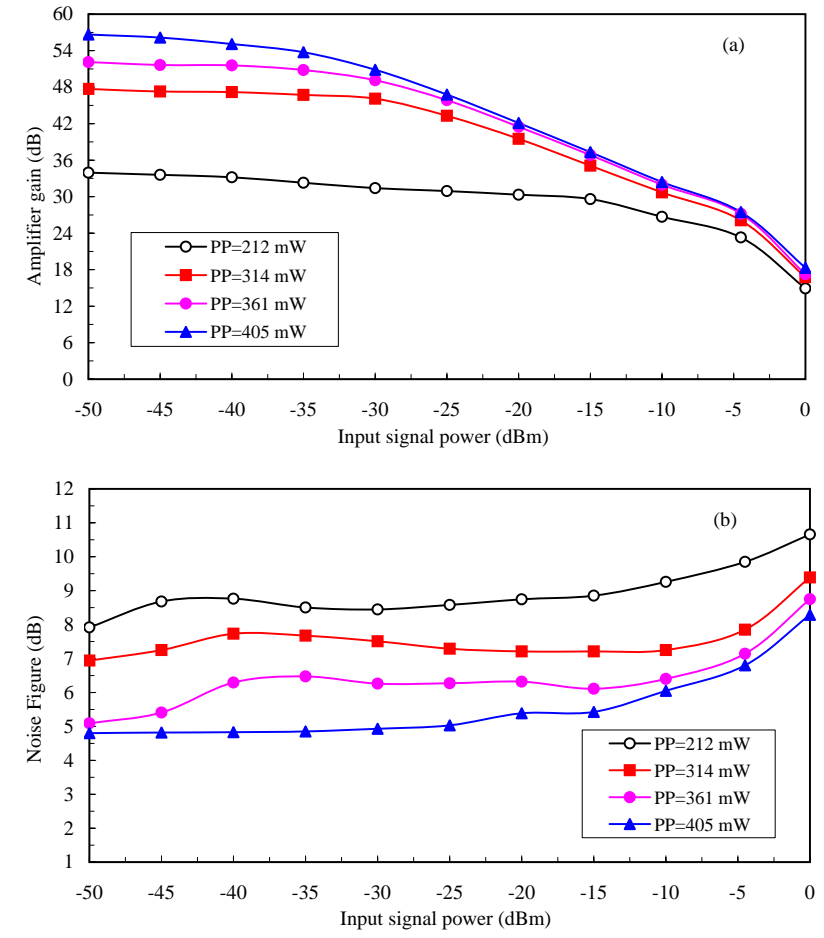

FIG. 4 (a) Amplifier gain and (b) noise figure against input signal power at $1570 \mathrm{~nm}$ wavelength with different pump powers.

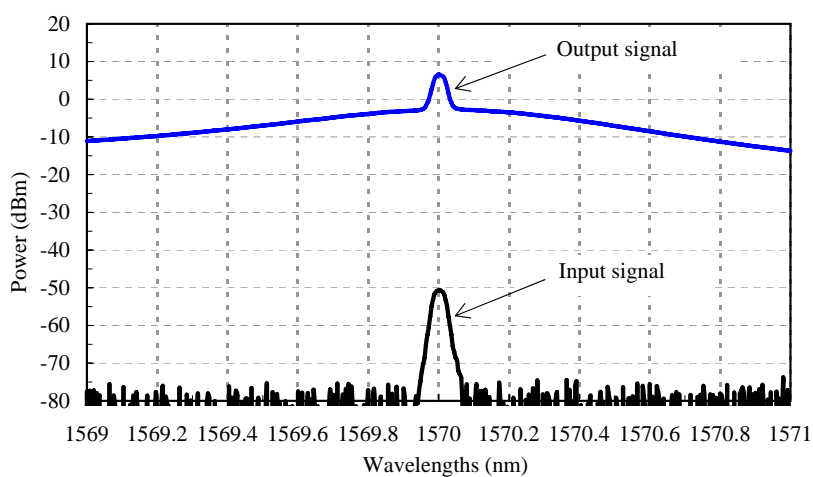

FIG. 5 Spectrum of the input signal and output signal of the amplifier at $405 \mathrm{~mW}$ pump power.

nal with $980 \mathrm{~nm}$ pump power of $405 \mathrm{~mW}$. The resolution of the OSA was set at $0.05 \mathrm{~nm}$ with span of $2 \mathrm{~nm}$.

To prove the enhancement of the amplifier gain due to the use of optical filter, we study the performance of the amplifier with TBPF and C/L coupler and without optical filter. The main function of the TBPF or C/L coupler is to suppress the backward C-band ASE noise from interning the second stage of the amplifier structure. Thus, the gain saturation due the C-band ASE noise will be eliminated. Figure 6 shows the amplifier gain against input signal power with and without filtering teaching at $405 \mathrm{~mW}$ of EDF pump power. At low input signal of $-50 \mathrm{dBm}$, the amplifier gain is improved to about $6 \mathrm{~dB}$ with $\mathrm{C} / \mathrm{L}$ band coupler and to about $13 \mathrm{~dB}$ with TBPF. The measured gain shows significant improvement compared to amplifier structure without C-band ASE suppression for input signal below $-25 \mathrm{dBm}$. The maximum gain obtained from 


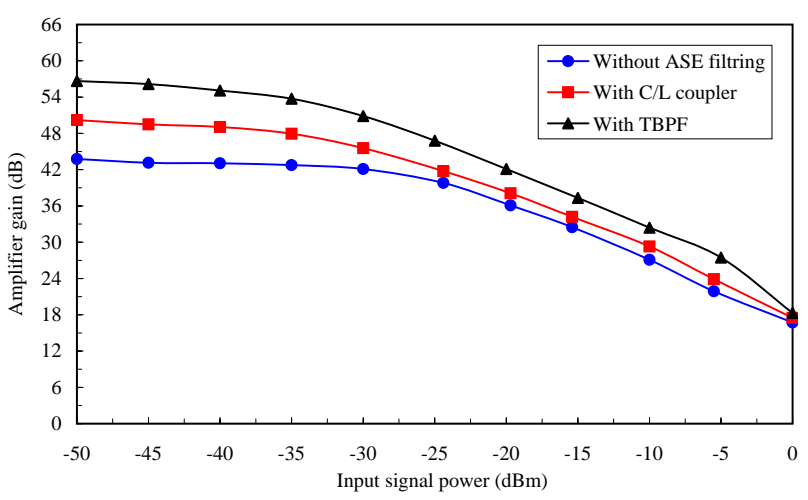

FIC. 6 Amplifier gain against input signal power at $1570 \mathrm{~nm}$ wavelength with and without ASE filtering at $405 \mathrm{~mW}$ pump power.

the experiment is $56.6 \mathrm{~dB}$ with noise figure of about $4.8 \mathrm{~dB}$ at $1570 \mathrm{~nm}$ wavelength with EDF pump power of $405 \mathrm{~mW}$.

\section{CONCLUSION}

We have studied the performance of triple-pass L-band EDFA using short-length highly-doped erbium rare-earth material with a single pump source for different input signal powers, signal wavelengths and pump powers. High gain with low noise figure optical amplifier is achievable using a single pump source and short gain medium. By including an optical filter between the amplifier stages, we obtain a high signal gain of $56.6 \mathrm{~dB}$ with noise figure less than $4.8 \mathrm{~dB}$. The use of $\mathrm{TBPF}$ in the correct position shows significant improvement in the amplifier gain compared to amplifier structure without TBPF. A gain improvement of $13 \mathrm{~dB}$ is achieved at low input signal of $-50 \mathrm{dBm}$.

\section{ACKN OWLEDGEMENT}

The research leading to these results has received research project grant funding from the Research Council of the Sultanate of Oman Research Grant Agreement No [ORG SU ICT 11 002]. The authors also acknowledge the support from Sohar University, Oman.

\section{References}

[1] E. Desurvire, J. R. Simpson, and P. C. Becker, "High-gain Erbium doped traveling-wave fiber amplifier," Opt. Lett. 12, 888-890 (1987).

[2] Y. Sun, J. W. Sulhoff, A. K. Srivastava, J. L. Zyskind, T. A. Strasser, J. R. Pedrazzani, C. Wolf, et al., "80 nm ultra-wide band erbiumdoped silica fiber amplifier," Electron. Lett. 33(23), 1965-1967 (1997).

[3] S. Hwang, K. W. Song, H. J. Kwon, J. Koh, Y. J. Oh, and K. Cho, "Broad band Erbium doped fiber amplifier with double-pass configuration," IEEE Photonic. Tech. L. 13(12), 1289-1291 (2001).

[4] C. H. Yeh, C. C. Lee, and S. Chi, "120 nm bandwidth erbium doped fiber amplifier in parallel configuration," IEEE Photonic. Tech. L. 17, 1055-1077 (2004).
[5] I. Yamashita, K. Shimoura, S. Seikai, and T. Fukuoka, "Er ${ }^{+3}$ doped fiber amplifier operating at wavelength of 1.55 and $1.60 \mu \mathrm{m}$," Electron. Lett. 32(12), 1102-1103 (1996).

[6] J. Nilsson, S. Y. Sun, S. T. Hwang, J. M. Kim, and S. J. Kim, "Longwavelength erbium-doped fiber amplifier gain enhanced by ASE end-reflectors," IEEE Photonic. Tech. L. 10, 1551-1553 (1998).

[7] M. A. Mahdi, and H. Ahmad, "Gain enhanced L-band $\mathrm{Er}^{3+}$-doped fiber amplifier utilizing unwanted backward ASE," IEEE Photonic. Tech. L. 13, 1067-1069 (2001).

[8] J. T. Ahn, M. Y. Jeon, and K. H. Kim, "Two stage reflective type erbium doped fiber amplifier with enhanced noise figure characteristics," Opt. Commun. 197(03) 121-125 (2001).

[9] H. Meng, W. Gao, Y. Liu, H. Zhang, C. Zhao, S. Yuan, X. Dong, et al., "The gain and noise figure improvement of reflection L-band erbium-doped fiber amplifier," Opt. Commun. 228, 85-89 (2003).

[10] H. B. Choi, J. M. Oh, and D. Lee, "Simple and efficient L-band erbiumdoped fiber amplifiers for WDM networks," Opt. Commun. 213, 63-66 (2002).

[11] L. L. Yi, L. Zhan, J. H. Ji, Q. H. Ye, and Y. X. Xia, "Improvement of gain and noise figure in double-pass L-band EDFA by incorporating a fiber Bragg grating," IEEE Photonic. Tech. L. 16, 1005-1007 (2004).

[12] S. W. Harun, and H. Ahmad, "Low noise double pass L-band erbium-doped fiber amplifier," Opt. Laser Technol. 36(3), 245-248 (2004).

[13] T. C. Liang and S. Hsu, "The L-band EDFA of high clamped gain and low noise figure implemented using fiber Bragg grating and double-pass method," Opt. Commun. 281, 1134-1139 (2008).

[14] B. Bouzid, M. B. Ali, and M. K. Abdullah, "High gain EDFA design using double pass amplification with bandpass filter," IEEE Photonic. Tech. L. 15(9), 1195-1197 (2003).

[15] S. W. Harun, N. Tamchek, P. Poopalan, and H. Ahmad, "Doublepass L-band EDFA with enhanced noise figure characteristics," IEEE Photonic. Tech. L. 15, 1055-1057 (2003).

[16] A. Altuncu, and A. Basgumus, "Gain enhancement in L-band Ioop EDFA through C-band signal injection," IEEE Photonic. Tech. L. 17(7), 1402-1404 (2005).

[17] M. A. Mahdi, and S. J. Sheih, "Low-noise 1480-nm pumped L-band erbium-doped fiber amplifiers incorporating a bypass isolator," Opt. Commun. 237, 295-299 (2004).

[18] R. Anthony, R. Lahiri, and S. Biswas, "Gain clamped L-band EDFA with forward-backward pumping scheme using fiber Bragg grating," Optik 125(11), 2463-2465 (2014).

[19] M. A. Mahdi, K. A. Khairi, B. Bouzid, and M. K. Abdullah, "Optimum pumping scheme of dual-stage triple pass erbium doped fiber amplifier," IEEE Photonic. Tech. L. 16(2), 419-421 (2004).

[20] Q. Z. Xuan, H. S. Ling, Z. X. Liang, and S. Fang, "A novel 3-stage structure for a low-noise, high-gain and gain-flattened L-band erbium doped fiber amplifier," J. Zhejiang Univ.-Sc. 5(9), 1130-1134 (2004).

[21] C. F. Su, and L. Wang, "Gain enhancement of L-band EDFA by using residual pump power in a three-stage configuration," 0 pt. Commun. 416, 412-416 (2007).

[22] N. Md. Yusoff, A. F. Abas, S. Hitam, and M. A. Mahdi, "Dual-stage Lband erbium-doped fiber amplifier with distributed pumping from single pump laser," Opt. Commun. 285(6), 1383-1386 (2012). 